\title{
Imaging Spectrum of Chronic Recurrent Multifocal Osteomyelitis Case Report: An Unusual Cause of Episodic Pain
}

\author{
Maryam Gul ${ }^{1}$, Abbas Chaudhry², Mubashir Sheikh², Amaan Kazi², Nina Haq ${ }^{2}$ and Ammar Chaudhry*3 \\ ${ }^{1}$ Assistant Professor of Rheumatology, City of Hope National Cancer Center, Beckman Research Institute, USA \\ ${ }^{2}$ Precision Imaging Lab, City of Hope National Cancer Center, Beckman Research Institute, USA
}

${ }^{3}$ Director of Precision Imaging Lab, Assoc. Director of Radiology and Imaging Informatics, City of Hope National Cancer Center, Beckman Research Institute, USA

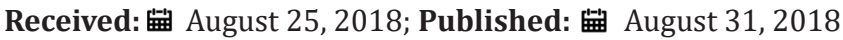

*Corresponding author: Ammar Chaudhry, Director of Precision Imaging Lab, Assoc. Director of Radiology and Imaging Informatics, City of Hope National Cancer Center, Beckman Research Institute, 1500 East Duarte Road, Duarte, CA 91010, USA

\section{Abstract}

Background: Chronic Recurrent Multifocal Osteomyelitis (CRMO) is a rare auto-inflammatory condition characterized by relapsing and remitting episodic bone pain most commonly affecting epiphyseal and diaphyseal regions of long bones. We present an unusual case of CRMO presenting as recurrent left lower quadrant pain and bilateral upper extremity pain.

Case Presentation: A 30-year-old Ethiopian female presented to the emergency department with intermittent worsening left lower quadrant pain and bilateral upper extremity pain. Past medical history is significant for sickle cell anemia. Physical exam including vital signs were unremarkable except for tenderness to palpation in bilateral upper extremity and left lower quadrant (posterior greater than anterior). Complete blood count revealed only mild anemia (Hemoglobin $10.1 \mathrm{mg} / \mathrm{dL}$ ), but was otherwise unremarkable, as was the comprehensive metabolic panel. Erythrocyte sedimentation rate (ESR) and C-reactive protein (CRP) were elevated at $71 \mathrm{~mm} / \mathrm{hr}$ and $12.3 \mathrm{mg} / \mathrm{L}$.

Conclusion: In patients with episodic extremity and abdominal pain, CRMO should be considered, especially in the absence of stigmata of infectious process or inflammatory bowel disease. The diagnosis of CRMO can be suggested by osteosclerosis on imaging studies and confirmed with biopsy. CRMO is usually treated with immunosuppressive therapy (glucocorticoids and interferon gamma) and NSAIDs. In our case, patient was successfully discharged to home on oral prednisone and NSAIDs (non-steroidal anti-inflammatory drugs). On follow-up visit two weeks after discharge, patient noted near complete resolution of her symptoms without interval relapse.

Keywords: Chronic; Osteomyelitis; Diagnosis; Auto-Inflammatory; Extremity; Imaging; Episodic; Metaphysis; Neoplasm; Osteomyelitis

Abbreviations: CRMO: Chronic Recurrent Multifocal Osteomyelitis; ESR: Erythrocyte Sedimentation Rate; CRP: C-Reactive Protein; LDH: Lactate Dehydrogenase; NSAID: Nonsteroidal Anti-Inflammatory Drug; MRI: Magnetic Resonance Imaging

\section{Background}

Chronic Recurrent Multifocal Osteomyelitis (CRMO) is an uncommon cause of diffuse osteosclerosis seen most commonly in the pediatric and young adult population, which is characterized by relapsing and remitting bone pain. Most common clinical symptoms include episodic pain of the involved osseous structures. Occasionally, if involved osseous structures are in the thorax, abdomen or pelvis, symptoms can be misinterpreted to be arising from the visceral structures. In CRMO, metaphysis is the most common region and tibia is the most common structure involved. Here, we describe a rare case of CRMO as continuous pain in the lower quadrant (involvement of the iliac bone) and bilateral upper extremity.

\section{Case Presentation}

A 30-year-old Ethiopian female presented to the emergency department with intermittent worsening left lower quadrant pain and bilateral upper extremity pain. Physical exam including vital signs were unremarkable except for tenderness to palpation in bilateral upper extremity and left lower quadrant (posterior greater than anterior). Complete blood count revealed only mild anemia (Hemoglobin $10.1 \mathrm{mg} / \mathrm{dL}$ ), but was otherwise unremarkable, as was the comprehensive metabolic panel. Erythrocyte sedimentation rate (ESR) and C-reactive protein (CRP) were elevated at $71 \mathrm{~mm} / \mathrm{hr}$ and $12.3 \mathrm{mg} / \mathrm{L}$. The patient did not have hemoglobinuria, avascular 
necrosis, splenic infarcts, and significant drop in her hemoglobin/ hematocrit, or other non-osseous stigmata to suggest this process could be from acute on chronic bone infarct in this patient with history of sickle cell anemia. Past medical history is significant for sickle cell anemia. Patient had normal LDH, no leukopenia/lymphopenia and no clinical signs (e.g. weight loss, anorexia, etc.) to suggest neoplasm (lymphoma, mastocytosis, etc)

The patient had no history of seizures, developmental disorder, angiofibromas, or other clinical findings to suggest tuberous sclerosis. Given patient's age and prior history of upper extremity bone pain, chronic recurrent osteomyelitis was included in the differential diagnosis. Abdominal radiograph was performed which demonstrated ill-defined areas of sclerosis in the left ilium (Figure 1). Radiograph of left upper extremity (Figure 2) demonstrated diffuse endosteal sclerosis of the left humerus with central lucencies. Due to worsening left upper extremity pain, patient underwent CT of her left upper extremity which demonstrated diffuse endosteal sclerosis, mild cortical expansion with prominent intramedullary lucency in the humeral head and diaphysis. There differential diagnosis based on patient's age and initial imaging findings was broad including infectious osteomyelitis, chronic recurrent multifocal osteomyelitis, systemic mastocytosis, lymphoma, leukemia, multiple osseous bone infarct and tuberous sclerosis. Given the broad differential and worsening pain, patient underwent MRI of the left upper extremity to further characterize the lesion and narrow the differential diagnosis. MRI reveal diffuse T1 hypointense T2 hyperintense signal with corresponding heterogeneous post-contrast enhancement (Figure 3).

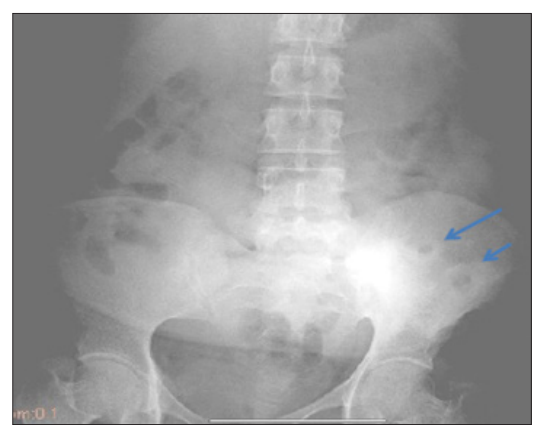

Figure 1: AP view of the lower abdomen, there are sclerotic lesions in the left ilium.

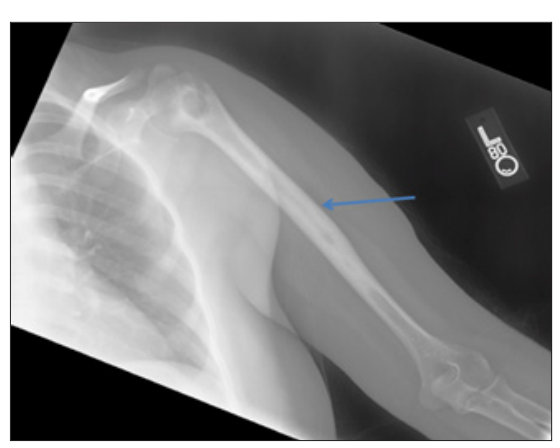

Figure 2: X-ray of the symptomatic area reveals cortical lesion with diffuse sclerosis.

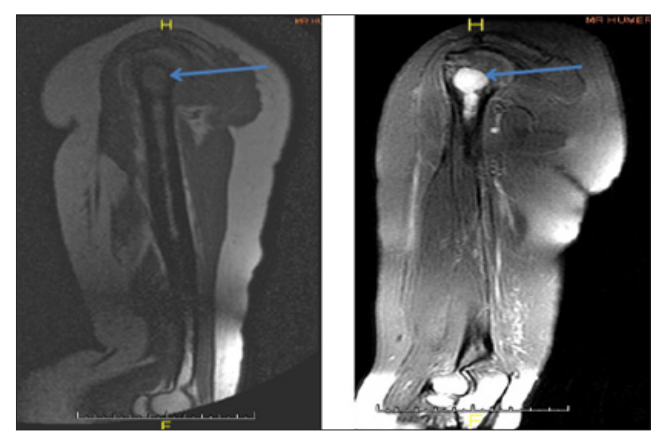

Figure 3: MRI of the left humerus 9(a) T1 SAG, (b) T2 SAG FSE Fat Sat reveal abnormal thickening of the left humeral cortex extending from the proximal metaphysis due to the distal diaphysis. There are foci of abnormal high fluid signal (arrow) within the medullary cavity of the humeral shaft extending into the humeral head and distal humeral diaphysis.

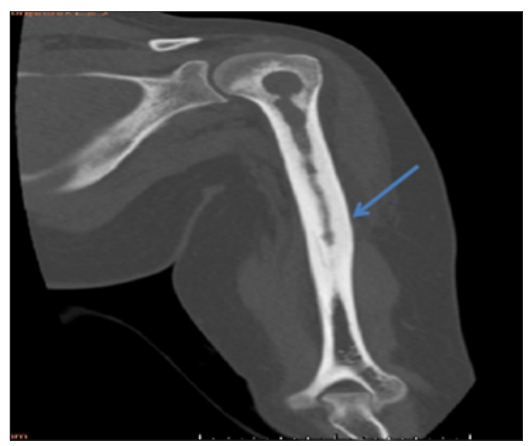

Figure 4: CT of the left upper extremity revealed intramedullary lucency in the humeral head and diaphysis with endosteal sclerosis and diffuse thickening of the humeral cortex with mild cortical expansion.

Patient underwent CT-guided core biopsy (Figure 4) of the left humerus using an 11-gauge biopsy needle. Specimens were sent for histopathologic evaluation, cytology, gram stain and culture. Histologic evaluation revealed many polymorphonuclear leukocytes, multinucleated giant cells, with some plasma cells, lymphocytes and histiocytes. There were no infectious organisms, mast cells or basophils identified on biopsy. The lack of mast cells and basophils along with absence of dermatosis excludes systemic mastocytosis. Absence of malignant cells and atypical lymphoid tissue excluded lymphoma and other potential malignant process which may cause osteoblastic metastases. Gram stain, cultures and PCRs (polymerase chain reaction) were negative for typical and atypical organisms. Interferon-gamma release assays were negative for tuberculosis. In light of this data along with imaging findings, the diagnosis of CRMO was confirmed.

On initial presentation and imaging findings, patient was empirically treated with broad spectrum IV antibiotics, intravenous (IV) glucocorticoids (62 mg methylprednisolone every 12 hours) and pain controlled with naproxen with tramadol administered on as needed basis. Upon exclusion of infection and histologic findings suggestive of CRMO, patient was treated with IV methylprednisolone for 7 days during which patient noted significant improve- 
ment in her abdominal and extremity pain. On follow-up visit two weeks after discharge, patient noted near complete resolution of her symptoms without interval relapse.

\section{Discussion and Conclusions}

Chronic Recurrent Multifocal Osteomyelitis (CRMO) is a rare auto-inflammatory condition characterized by relapsing and remitting episodic bone pain secondary to sterile inflammatory foci. CRMO belongs to a group of disorders sharing the common feature of chronic non-infectious osteomyelitis [1]. Some of these entities include synovitis, acne, pustulosis, hyperostosis and osteitis syndrome (SAPHO), Majeed syndrome, deficiency of interleukin-1 receptor antagonist, and cherubism. CRMO is considered an "orphan disease", affecting less than 200,000 individuals within the United States. Estimated prevalence is less than $1 / 1,000,000$ although this figure may be underestimated due to the difficulty in disease recognition [1]. CRMO primarily affects children and adolescents with mean age of diagnosis at 10 years, although reported cases have ranged from 6 months to 55 years in age [2-6]. A slight female predilection has been described [5].

The most salient feature of CRMO is bone pain predominating in the epiphysis and metaphysis of long bones. Pain is typically episodic, nocturnal, and insidious and may be accompanied by local erythema and soft tissue edema. Systemic signs such as low-grade fever, weight loss, and generalized malaise have also been described. The most common sites of involvement are as follows, in order of decreasing frequency: distal tibia, pelvis, proximal femur, clavicle, and calcaneus [3]. These sites are symmetric in up to $25 \%$ of patients. In particular, involvement of the clavicle and other even less commonly involved areas such as the sternum and manubrium are highly suggestive of CRMO. Except for trauma, CRMO is the most common non-neoplastic process involving the clavicle in patients younger than 20 years old, and it is the most common disease to involve the medial third of the clavicle in all age groups [6]. Although rare, vertebral CRMO has been described and may result in vertebra plana [9]. Extra-skeletal manifestations include psoriasis, palmoplantar pustulosis, Crohn's disease, acne, and Sweet's syndrome $[1,6,7]$. Although considered a disease of childhood, reports have shown that episodic recurrences persist greater than 10 years in up to $59 \%$ of cases.

In addition to chronic pain, long term consequences of CRMO include osseous deformities such as limb length discrepancy. Treatment for CRMO usually involves immunosuppressive therapy, including glucocorticoids and interferon gamma. Studies have shown that bisphosphonate therapy has also been effective in decreasing bone pain and improving remodeling by restoring vertebral body height when the spine is involved $[1,8]$. TNF-alpha blockers have also been effective in treating cases with vertebral involvement $[1,2]$. Bone biopsy is often performed to exclude infectious osteomyelitis and neoplastic processes. Upon histopathologic evaluation, CRMO lesions demonstrate nonspecific inflammatory changes with granulocytic infiltration. The early inflammatory stages are marked by a predominance of polymorphonuclear leukocytes as well as osteoclastic bone resorption with or without multinucleated giant cells $[4,6]$. As the disease progresses there is infiltration by lympho- cytes, plasma cells, histioctyes, and occasionally granulomata. Biopsy specimens often demonstrate temporal heterogeneity with a mix of acute, subacute, and chronic histopathologic findings. In contrast to infectious osteomyelitis, bone cultures are negative.

The radiographic appearance of CRMO lesions range from can purely osteolytic, osteolytic with a sclerotic border, mixed lytic and sclerotic, and purely sclerotic $[4,6]$. Initial imaging findings include lytic metaphyseal lesions adjacent to the growth plate that progressively become thin and sclerotic in approximately 1-2 weeks $[2,3,6]$. There is subsequent sclerosis and hyperostosis over time as the inflammation extends to the cortex. MR imaging is useful in both diagnosis and surveillance of disease. The acute phase of disease demonstrates marrow edema as evidenced by T1-weighted hypointense and T2-weighted hyperintense signal. Associated periostitis, physeal involvement, and surrounding soft tissue inflammation can also be evaluated with MR imaging. It has been noted that up to $30 \%$ of CRMO cases demonstrate articular involvement including clinically occult joint effusions, synovial thickening, cartilaginous and subchondral bone destruction [6]. The presence of large fluid collection, abscess, fistulous tract, or sequestrum makes infectious osteomyelitis more likely than CRMO $[1,7,9]$.

\section{Acknowledgment}

The authors are grateful for both the patient's contribution and IRB exemption to allow publishing of case findings.

\section{Availability of Data and Materials}

Data is available upon request to the corresponding author Dr. Ammar Chaudhry

\section{Author's Contributions}

MG and AaC conceptualized and designed the case report, collected patient data relevant to the case report and drafted the initial manuscript. AbC critically reviewed and revised the initial draft of the manuscript and added important intellectual discussion points identified from literature review. This was followed by second line of review and revisions by $\mathrm{AK}$ and $\mathrm{NH}$ who also critically reviewed and revised the initial manuscript in accordance with the journal requirement to its current form. All authors approved the final manuscript as submitted and agree to be accountable for all aspects of the work.

\section{Ethics Approval and Consent to Participate}

This case study was reviewed by the institutional review board and approved by the IRB. Given nature of the anonymized case presentation, this case report for approved for exemption for patient consent.

\section{Consent for Publication}

For this case report, no consent was required as per institutional review board policies.

\section{References}

1. Costa Reis P, Sullivan KE (2013) Chronic recurrent multifocal osteomyelitis. Journal of Clinical Immunology 33: 1043-1056. 
2. Buck FM, Treumann TC, Winkier H, Strobel K (2007) Chronic recurrent multifocal osteomyelitis (CRMO) with symmetric involvement of both femora: x-ray, bone scinitgram, and MR imaging findings in one case. Journal of Magnetic Resonance Imaging 26: 422-426.

3. Wipff J, Adamsbaum C, Kahan A (2011) Chronic recurrent multifocal osteomyelitis. Joint Bone Spine 78: 555-560.

4. Girschick HJ, Huppertz H, Harmsen D, krauspe R, Papadopoulos T, et al. (1999) Chronic recurrent multifocal osteomyelitis in children: diagnostic value of histopathology and microbial testing. Human Pathology 30(1): 59-65.

5. Falip C, Alison M, Boutry N, Cotton A, Azoulay R et al. (2013) Chronic recurrent multifocal osteomyelitis (CRMO): a longitudinal case series review. Pediatric Radiology 43(3): 355-375.

ISSN: 2574-1241

DOI: $10.26717 / B J S T R .2018 .08 .001676$

Ammar Chaudhry. Biomed J Sci \& Tech Res

(C) This work is licensed under Creative

Submission Link: https://biomedres.us/submit-manuscript.php
6. Khanna G, Sato T, Ferguson P (2009) Imaging of chronic recurrent osteomyelitis. Radiographics 29(4): 1159-1177.

7. Thakur U, Blacksin M, Beebe K, JC Neilson, Gino T, et al. (2012) Synovitis, acne, pustulosis, hyperostosis and osteitis (SAPHO) and chronic recurrent multifocal osteomyelitis (CRMO): role of imaging in diagnosis. Radiography 18(3): 221-224.

8. Gleeson H, Wiltshire E, Briody J, Hall J, Cowell C, et al. (2008) Childhood chronic recurrent multifocal osteomyelitis: pamidronate therapy decreases pain and improves vertebral shape. The Journal of Rheumatology 35(4): 707-712.

9. Demharter J, Bohndorf K, Michl W, Voqt H (1997) Chronic recurrent multifocal osteomyelitis: a radiological and clinical investigation of five cases. Skeletal Radiology (26): 579-588.

$\begin{array}{ll}\text { BIOMEDICAL } & \text { Assets of Publishing with us } \\ \text { RESEARCHES } & \text { - Global archiving of articles } \\ & \text { - Immediate, unrestricted online access } \\ & \text { - Rigorous Peer Review Process } \\ & \text { - Authors Retain Copyrights }\end{array}$

